\title{
Effect of applying resistance in various directions on lower extremity muscle activity and balance during squat exercise
}

\author{
Jung-Eun Song ${ }^{a}$, Ho-Suk Choi ${ }^{b}$, Won-Seob Shin ${ }^{a, b}$ \\ aDepartment of Physical Therapy, Graduate School of Health and Medical, Daejeon University, Daejeon, Republic of Korea \\ ${ }^{\mathrm{b}}$ Department of Physical Therapy, College of Health and Medical Science, Daejeon University, Daejeon, Republic of Korea
}

Objective: The purpose of this study was to investigate the effects of resistance applied in various directions on lower extremity muscle activity and balance during squat exercise performance.

Design: Cross-sectional study.

Methods: Forty-one adults (19 males and 22 females) agreed to the study purpose and procedures. All subjects randomly performed squat exercises with an intensity of overcoming $10 \%$ of body weight resistance pulled forward, backward and general squats with $60^{\circ}$ of knee joint flexion. Electromyography was used to measure muscle activity of the vastus medialis oblique (VMO), rectus femoris (RF), vastus lateralis oblique (VLO), biceps femoris (BF), and semitendinosus (ST) muscles. The Wii Balance Board was used to assess balance during the three-method squat operation. Each operation was measured three times for 10 seconds.

Results: There were significant differences in muscle activities of the VMO, RF, VLO, ST and balance ability with the application of three directions of resistance $(p<0.05)$. Post hoc comparisons revealed that squats performed with resistance pulled in the backward direction resulted in higher VMO, RF and VLO activity than with the resistance placed in a pulled forward direction $(p<0.05)$. In the ST, resistance applied in the pulled forward direction showed greater muscle activity compared to the pulled backward direction $(p<0.05)$. With balance, squats performed with resistance pulled in the forward direction showed greater muscle activity than with resistance applied in the pulled backward direction and during general squat performance $(p<0.05)$.

Conclusions: In this study, squat exercises performed with resistance applied in the direction of pulling backwards was found to be the most effective in improving quadriceps muscle strength and balance. It is effective to provide resistance that is placed in the forward when it is difficult to perform a general squat due to weakness of the quadriceps.

Key Words: Electromyography, Exercise, Muscles, Postural balance

\section{Introduction}

Due to recent improvements in living standards, leisure time has become more important, and since the interest in health has increased, those who enjoy sports and leisure activities are increasing in number. As a result, body damage is also increasing. Therefore, as the importance of management of injuries caused by exercise is newly recognized, it is emphasized that there is need for active rehabilitation for prevention of injury as well as for quick recovery [1].

Knee joint damage has increased during various sports injuries, accounting for $14 \%-16 \%$ of total musculoskeletal injuries, which is the second most common injury following ankle joint injury in total injuries. If immediate treatment is not provided, knee joint instability may occur and activity levels may be deteriorated due to the limitation of daily life

Received: 3 May, 2019 Revised: 27 May, 2019 Accepted: 28 May, 2019

Corresponding author: Won-Seob Shin (ORCID https://orcid.org/0000-0002-6515-7020)

Department of Physical Therapy, College of Health and Medical Science, Daejeon University, 62 Daehak-ro, Dong-gu, Daejeon 34520, Republic of Korea Tel: 82-42-280-2294 Fax: 82-42-280-2295 E-mail: shinws@dju.kr

(c) This is an Open-Access article distributed under the terms of the Creative Commons Attribution Non-Commercial License (http://creativecommons.org/licenses/ by-nc/4.0) which permits unrestricted non-commercial use, distribution, and reproduction in any medium, provided the original work is properly cited.

Copyright $\odot 2019$ Korean Academy of Physical Therapy Rehabilitation Science 
[2-4].

Recently, it has been found that conservative treatment is effective for persons with knee injuries [5]. The most commonly used interventions are quadriceps strengthening exercises.

Closed kinetic chain squat exercise is a type of resistance exercise that usesmultiple joints at the same time [6]. This type of closed-chain exercise is effective and safe because it provides joint stability through co-contraction of the quadriceps and hamstring muscles and provides less stress to the knee joints [7,8]. Establishing a proper quadriceps to hamstring ratio throughout the squat movement plays an important role in preventing knee injury by increasing the stability of the knee joint and reducing stress placed onto the joint [9]. Squat exercises are appropriate for thigh muscle formation and quadriceps muscle strengthening since it supports the body weight, which promotes the pattern of muscle recruitment. It is also an efficient exercise for improving proprioception [10].

Squat exercises play an important role in everyday life, such as walking, balancing, and running [11]. Squat exercises can enhance one's ability to maintain attitudes to a variety of external environments [12]. Also, squat exercises improve muscle strength and power, which improves balance ability by reducing the sway velocity [13].

Other prior studies have provided resistance on the inside and outside of the lower extremity using a non-quantitative tool, such as a Swiss ball or theraband during the squat exercise.

In addition, backward resistance using a theraband during a squat exercise combined with external rotation of the hip joint can selectively strengthen the vastus medialis oblique (VMO) muscle [14]. Squat exercise, with resistance applied in the anterior-lateral $45^{\circ}$ direction, increased the $\mathrm{VMO} /$ vastus lateralis oblique (VLO) ratio of muscle activity [15]. Resistance applied to the hip adduction direction during squatting significantly increased the muscle activity of the VLO [16].

However, no studies have been conducted to measure muscle activity and balance ability while providing quantitative resistance in the forward and backward direction.

The purpose of this study was to investigate the quadriceps and hamstring muscle activity and balance ability when providing quantitative resistance in the forward and backward direction during squat performance.

\section{Methods}

\section{Participants}

Forty-one adults from Daejeon University in Daejeon were enrolled as subjects for this study. All subjects participating in the study were provided with sufficient explanation prior to the start of the experiment.

The selection criteria for the subjects were those with no medical illnesses in the trunk and lower extremities, no difficulty in exercising, capable of performing squat exercises, and no open wounds on the electrode attachment site.

The exclusion criteria were those who could not perform $60^{\circ}$ of knee flexion during the squat exercise, inability to maintain a squat position when applying resistance, and currently receiving therapeutic intervention.

A pilot study was conducted to determine the sample size. The $\mathrm{G}^{*}$ power (version 3.1.9.2; Heinrich-Heine-Universität, Düsseldorf, Germany) was used for alpha 0.05, and the power of 0.9 was used for each sample. The effect size was 0.55 , and the sample size was calculated as 36 using $\mathrm{G}^{*}$ power program. Two of the dropouts occurred during the study. Therefore, a total of 41 subjects were recruited (Table 1).

After being fully explained about the purpose and process of the study, all subjects voluntarily decided to participate and they written informed consent. All the procedures and methods of this study have been approved by the Institutional Review Board of Daejeon University (IRB No. 1040647-201804-HR-019-03) and follows the principles outlined in the Declaration of Helsinki.

\section{Exercise procedure}

This study was performed by providing three squat exercise conditions (Figure 1). All subjects were randomly assigned to complete all three conditions. In this study, the arms were stretched forward, parallel with the floor. With the feet flat, the feet were spread to be shoulder-width apart. The arms were stretched forward, parallel with the floor. With the feet flat, the feet were spread to be shoulder-width

Table 1. Basic characteristics of participants

$(\mathrm{N}=41)$

\begin{tabular}{lcc}
\hline Characteristic & Average & Range \\
\hline Age $(\mathrm{y})$ & $22.15(1.54)$ & $19-26$ \\
Height $(\mathrm{cm})$ & $166.66(9.00)$ & $153-185$ \\
Weight $(\mathrm{kg})$ & $65.22(14.68)$ & $46-110$ \\
Sex (male/female) & $19 / 22$ & \\
\hline
\end{tabular}

Values are presented as mean (SD) or number only. 
apart. The knee did not cross the toes and the knee flexion angle was set at $60^{\circ}$ [17]. In order to align the body, the patella was directed to the second toe. The above posture was maintained according to the instructions of the research assistant [18]. Subjects were instructed to maintain the squat exercise posture for 10 seconds. To reduce the effects of fatigue, a 3-minute rest period between the exercise conditions was provided. Each exercise method was repeated three times and analyzed using mean values.

In the preliminary experiments of this study, squat exercises were performed with resistance corresponding to $5 \%, 10 \%$, and $15 \%$ of body weight. As a result, there was no significant difference in the results when $5 \%$ of body weight was provided and when $15 \%$ of body weight was provided, the squat position was not maintained. Thus, the amount of resistance provided $10 \%$ of body weight.

The resistance during the squat exercise was given as follows. First, a general squat exercise without resistance. Second, a resistance corresponding to $10 \%$ of body weight was provided from anterior to posterior via a tension meter connected with a strap at $1 / 3$ of the femur. Second, a resistance corresponding to $10 \%$ of body weight was provided from posterior to anterior via a tension meter connected with a strap at $1 / 3$ of the femur (Figure 2).

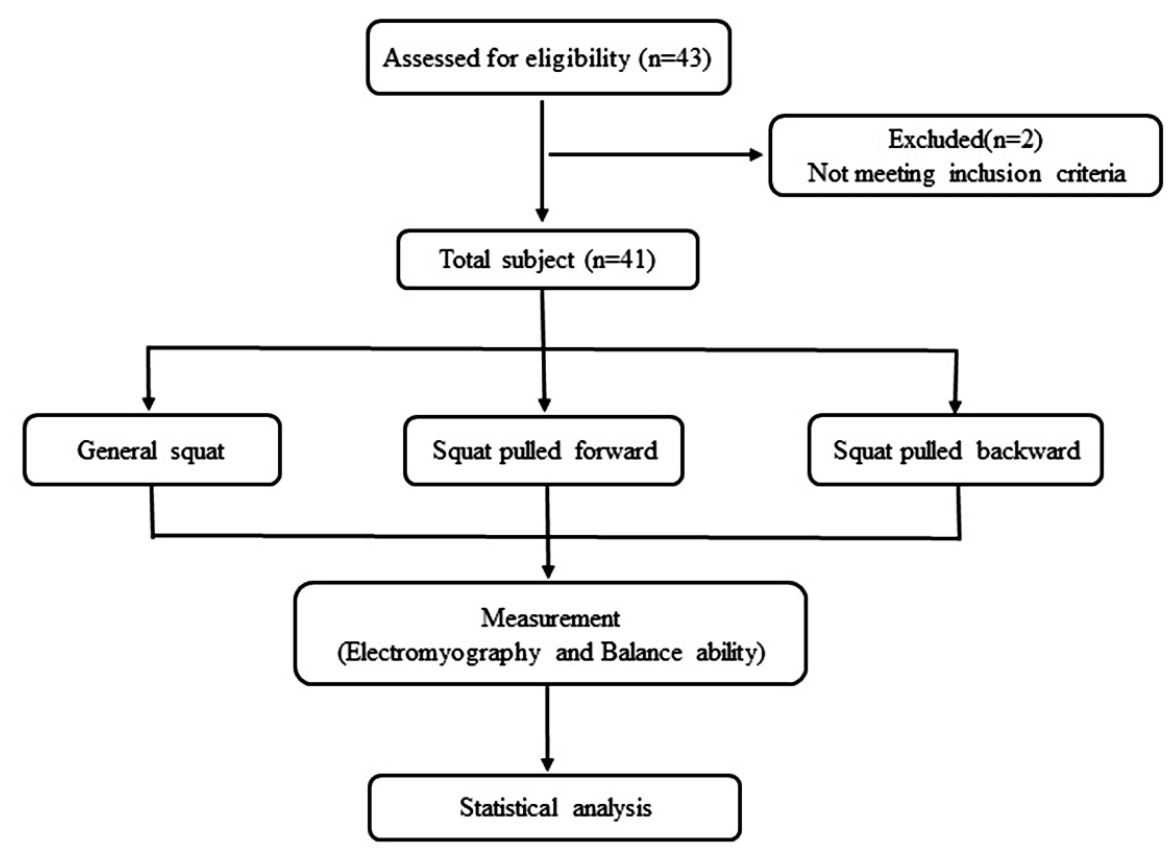

Figure 1. Flowchart of participants through the trial.

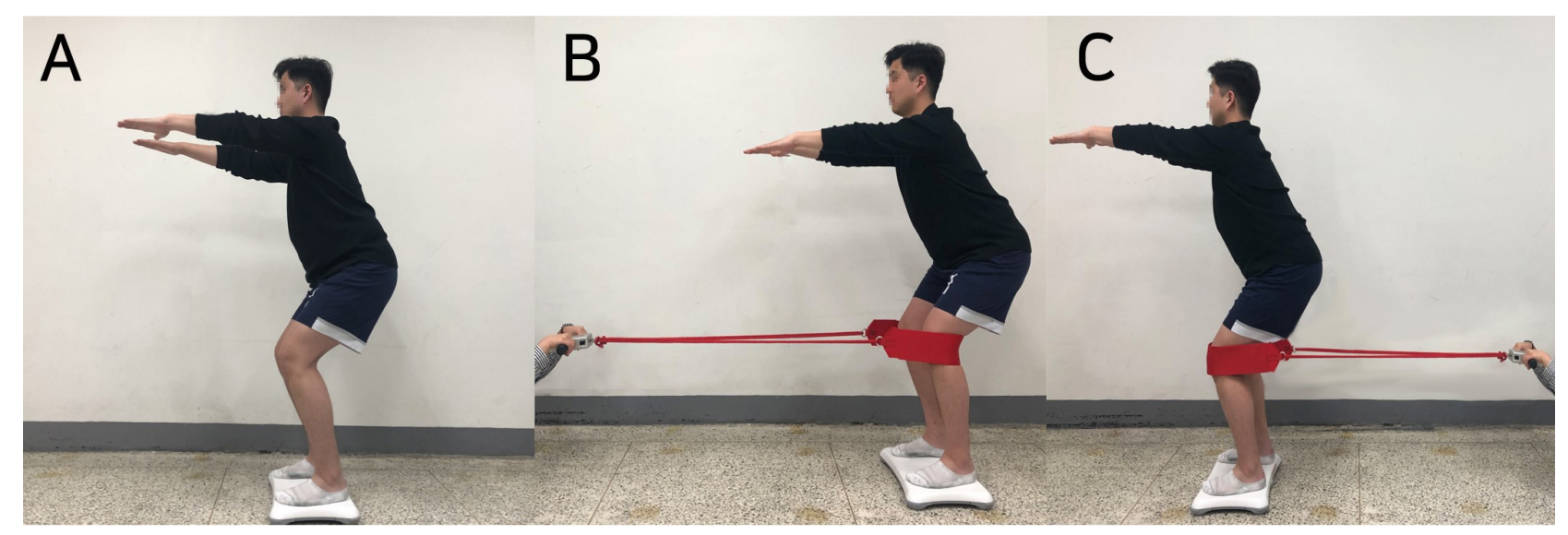

Figure 2. Resistance direction during squat. (A) General squat, (B) pulled forward squat, (C) pulled backward squat. 
Table 2. Comparison of mean muscle activity and balance ability $(\mathrm{N}=41)$

\begin{tabular}{lcccc}
\hline \multicolumn{1}{c}{ Variable } & General squat & Pulled forward squat & Pulled backward squat & $\mathrm{F}$ \\
\hline Muscle activity (\%MVIC) & & & & \\
VMO & $78.92(41.60)$ & $55.94(27.94)^{\mathrm{a}}$ & $80.13(40.85)^{\mathrm{b}}$ & $25.167^{* *}$ \\
RF & $87.44(36.49)$ & $62.52(27.66)^{\mathrm{a}}$ & $90.82(33.70)^{\mathrm{b}}$ & $43.317^{* *}$ \\
VLO & $123.06(60.39)$ & $99.31(53.28)^{\mathrm{a}}$ & $123.46(58.83)^{\mathrm{b}}$ & $9.663^{* *}$ \\
BF & $28.63(20.93)$ & $30.70(22.44)$ & $29.15(19.41)$ & 0.573 \\
ST & $29.52(21.43)$ & $32.85(23.00)$ & $27.97(17.86)^{\mathrm{b}}$ & $3.461^{*}$ \\
Balance ability & & & & \\
Sway distance (mm) & $44.16(11.93)$ & $42.68(8.36)^{\mathrm{a}}$ & $48.32(11.88)^{\mathrm{a}, \mathrm{b}}$ & $8.321^{* *}$ \\
Sway velocity (mm/s) & $4.42(1.19)$ & $4.26(0.84)^{\mathrm{a}}$ & $4.83(1.19)^{\mathrm{a}, \mathrm{b}}$ & $8.379^{* *}$ \\
\hline
\end{tabular}

Values are presented as mean (SD).

MVIC: maximum voluntary isometric contraction, VMO: vastus medialis oblique, RF: rectus femoris, VLO: vastus lateralis oblique, BF: biceps femoris, ST: semitendinosus.

${ }^{\mathrm{a}}$ Significant difference with general squat $(p<0.05)$. ${ }^{\mathrm{b}}$ Significant difference with pulled forward squat $(p<0.05)$.

$* p<0.05,{ }^{*} p<<0.01$

\section{Experimental equipment}

\section{Muscle activity measure and data processing}

To assess muscle activity during the squat exercise, surface electromyography (EMG, Myosystem 1400A; Noraxon Inc., Scottsdale, AZ, USA) was used. The sampling rate of the EMG signal was set to $1,000 \mathrm{~Hz}$ and the frequency bandwidth was set to 40 to $250 \mathrm{~Hz}$. All data was processed using the MyoReserach Xp 2.02 software (Noraxon Inc.).

The root mean square of the signal was calculated and normalized to the EMG data maximum voluntary contraction. The maximum isometric contraction value of each muscle was normalized by converting the average signal value of the peak value for 5 seconds except for the first and last second, leaving the middle 3 seconds to be used to calculate the \%maximum voluntary isometric contraction, which was performed three times. The surface electrodes were attached to the dominant muscle and parallel to the muscle fibers by the same experimenter. To attach the electrodes properly, foreign substances were removed by shaving and wiping the attachment site with alcohol. The location of the electrodes and all the details followed the guidelines of Cram et al. [19].

\section{Balance ability}

In order to measure the static balance ability during the squat exercise, we measured the sway velocity and sway distance using the Wii Balance Board (Nintendo, Kyoto, Japan). With both feet placed on the balance board the subjects performed the squat exercise. Balance ability was then measured when resistance was provided in various directions.
Balance ability was assessed by collecting data on the center of gravity of the $\mathrm{X}$ and $\mathrm{Y}$ axes during the squat exercise. This was measured three times for 10 seconds and the average value was used. The Balancia program (Balancia software ver. 2.0; Mintosys, Seoul, Korea) was used to analyze the collected data.

\section{Data and statistical analysis}

All materials were analyzed using the IBM SPSS Statistics ver. 22.0 (IBM Co., Armonk, NY, USA) was used. The general characteristics of the subjects were described by mean and standard deviation through descriptive statistics. One-way repteated measure ANOVA was used to compare the differences when providing resistance in three different directions during squat exercise, and a post-hoc test was used by Bonferroni correction. The statistical significance level was set at $p<0.05$.

\section{Results}

\section{Comparison of muscle activity}

The comparison in muscle activity is shown in Table 2 . When applying the three methods of squat exercise, muscle activity was statistically significantly different between quadriceps and semitendinosus (ST) muscles $(p<0.05)$. The quadriceps (VMO, RF, and VLO) showed higher muscle activity with resistance application compared to the normal squat exercise and pulled backward squat exercise. The ST showed higher muscle activity with resistance application compared to the than normal squat exercise and pulled forward squat exercise. 
In the post-hoc analysis, the quadriceps muscle activity was significantly different between general squat and pulled forward squat, and between pulled forward squat and pulled backward squat. The ST muscle activity was significantly different between the pulled forward squat and pulled backward squat $(p<0.05)$.

\section{Comparison of balance ability}

The comparison in balance ability is shown in Table 2 . Sway velocity and sway distance values were significantly different $(p<0.05)$. Balance ability was higher than the normal squat and the pulled backward squat. In the post-hoc analysis, balance ability was significantly different between the pulled backward squat and general squat, and between the pulled backward and pulled forward squat $(p<0.05)$.

\section{Discussion}

Since the squat exercise forces the body weight to be placed uniformly onto both legs, it is possible to improve muscle strength more effectively in a stable posture than other lower body strengthening exercises, such as lunge, and it is considered to be the most appropriate exercise that can strengthen the hip and proximal lower extremity muscles as well as strengthen the trunk muscles together. When a heavy load is overcome by the body, such as when performing a squat exercise, or when the body is suddenly loaded, the muscles around the trunk are co-activated to maintain posture and increase stability [20].

The purpose of this study was to investigate the effect of squat exercise when providing a resistance pulled forward and backward to maintain balance ability and the muscle activity of the proximal lower extremity.

A comparison of muscle activity and balance ability of the lower limbs according to three resistance directions, which are the general squat exercise without resistance, squat exercise with resistance applied from the back to the front, and squat exercise with resistance applied from the front to the back, muscle activity was significantly different in the VMO, rectus femoris (RF), VLO, and ST. The balance ability increased the value of the sway distance and velocity in the squat exercise with resistance provided in the backward direction $(p<0.05)$.

In a previous study, when resistance was provided in the direction of abduction of the hip joint during squatting, the muscle activity of the VMO was increased, and when resistance was provided in the direction of adduction of the hip joint during squatting, the muscle activity of the VLO was increased [16]. The VMO, RF, and VLO muscles located in front of the body showed a significant difference in muscle activity when resistance was provided in the pulled backward direction, and the ST muscle located at the back of the body showed a significant difference when resistance was applied in the pulled forward direction $(p<0.05)$.

The hamstring muscle plays a role in maintaining a stable element of the movement of the knee joint in daily life activity, and to relieve the pressure exerted internal and external pressure of the knee joint interacting with quadriceps [21]. In the standing posture, the muscle activity of the hamstring and the calf muscles increase when providing the same sway as the method of providing resistance from the back to the front [22]. This result is similar to the increase in muscle activity of the ST when resistance is applied in the pulled forward direction in this study. When ankle dorsiflexion is combined during the squat exercise, the activity of the biceps femoris (BF) muscle is increased due to instability [23]. In this study, the BF activity was not significantly different. It is thought that the BF on the back of the body is activated by the resistance pulled forward. Also, it is thought that the $\mathrm{BF}$ is activated by the pulled backward resistance, which is the most unstable environment.

The use of a visual feedback device placed at the front during squat exercise helps to maintain a stable balance with the increase in the muscle thickness of the quadriceps and transverse abdominis [24]. In this study, when resistance was applied in the pulled forward, the motion of the inspector providing the resistance acted as visual feedback to the subject. As a result, it is similar to the result of the reduction of the fluctuation sway distance and velocity.

Also, while squatting, the center of pressure was positioned behind the body. Providing resistance in the pulled forward direction has pulled forward the center of pressure located at the rear. Therefore, it is considered that an unstable result was obtained when the resistance applied in the pulled backward direction was provided.

As a result, it is recommended to provide resistance that is pulled backward during the squat exercise in order to increase balance ability, including selective strengthening of the quadriceps of the lower extremity muscles, which are known to be imptortant muscles in daily life and sports activities. Also, for the subjects who had difficulty performing the squat exercises due to weak quadriceps, it is effective to perform the squat exercise with the addition of resistance in the pulled forward direction, which had resulted in de- 
creased quadriceps muscle activity. In the case of the selective strengthening of the hamstrings, it is considered that providing resistance in the pulled forward direction during the squat exercise will promote positive muscle strengthening results.

The limitation of this study is that the is difficult to generalize the results of the study since it included healthy college students as subjects. The measurement of the muscles of the tibial bones, such as the tibialis anterior and the calf muscles affected during squat exercise was not performed. Also, resistance was provided only in the lower limb and the study did not focus on the effect of overall muscle activity. In future studies, it would be necessary to study the muscle activity of the trunk and other various muscles, and to examine the effects of applying resistance to other parts of the body in consideration of these limitations.

\section{Acknowledgements}

This work was supported by the National Research Foundation of Korea (NRF) grant funded by the Korea government (MSIP) (No. 2016R1C1B2010420).

\section{Conflict of Interest}

The authors declared no potential conflicts of interest with respect to the authorship and/or publication of this article.

\section{References}

1. Cowan SM, Bennell KL, Hodges PW, Crossley KM, McConnell J. Delayed onset of electromyographic activity of vastus medialis obliquus relative to vastus lateralis in subjects with patellofemoral pain syndrome. Arch Phys Med Rehabil 2001;82:183-9.

2. Kim ID, Lee SY, Kim PT, Park BC, Park HJ. A clinical study on ligamentous injury of the knee. J Korean Orthop Assoc 1984; 19:1087-95.

3. Yang JH, Cho TH, Yoo SC. A clinical study on ligamentous injury of the knee. J Korean Orthop Assoc 1988;23:383-92.

4. Han SW. Effect of rehabilitation exercise and neuromuscular electrical stimulation on visual analysis scale and on functional capacity performed for 8 weeks in a patient with patellofemoral pain syndrome. Phys Ther Korea 2004;11:33-42.

5. Park JT, Part YG. Manual of clinical orthpedics. 3rd ed. Seoul: Hyunmoon publishing Co.; 2002.

6. Salem GJ, Powers CM. Patellofemoral joint kinetics during squatting in collegiate women athletes. Clin Biomech (Bristol, Avon) 2001;16:424-30.

7. Wilk KE, Davies GJ, Mangine RE, Malone TR. Patellofemoral disorders: a classification system and clinical guidelines for non- operative rehabilitation. J Orthop Sports Phys Ther 1998;28: 307-22.

8. Lim SY, Lee WH. Effect of two types of muscle contraction training on muscle thickness, strength, and delayed onset of muscle soreness in persons with chronic stroke. Phys Ther Rehabil Sci 2018;7:154-63.

9. Kim JE, Kim HA, Lee JU, Jung HS, Cho UK, Choi HM, et al. Changes in the quadriceps-to-hamstring muscle ratio during wall squatting according to the straight leg raise test angle. Phys Ther Rehabil Sci 2019;8:45-51.

10. Selseth A, Dayton M, Cordova ML, Ingersoll CD, Merrick MA. Quadriceps concentric EMG activity is greater than eccentric EMG activity during the lateral step-up exercise. J Sport Rehabil 2000;9:124-34.

11. Chung HK. Study for squat scoring scale. J Korea Soc Phys Ther 2004;16:821-9.

12. McGill SM, Cholewicki J. Biomechanical basis for stability: an explanation to enhance clinical utility. J Orthop Sports Phys Ther 2001;31:96-100.

13. Kim SH, Kim MH, Kim HJ. Effects of jump rope and squat exercise on power and balance ability. J Korea Entertain Ind Assoc 2015;9:125-31.

14. Hammer WI. Functional soft-tissue examination and treatment by manual methods. Sudbury (MA): Jones and Bartlett Pub.; 2007.

15. Nam KS. Effect of the resistance direction by an elastic band on the $\mathrm{VMO} / \mathrm{VL}$ electromyographic activity ratio during dynamic squat exercise. J Korea Soc Phys Ther 2008;20:29-34.

16. Lee SE, Kim JW, Hwang BJ. The influence of applied resistance to hip joint during single limb squat on the muscle activation of qudriceps muscle in patellofemoral pain syndrome patient. Arch Orthop Sports Phys Ther 2016;12:31-42.

17. Escamilla RF, Fleisig GS, Zheng N, Lander JE, Barrentine SW, Andrews JR, et al. Effects of technique variations on knee biomechanics during the squat and leg press. Med Sci Sports Exerc 2001;33:1552-66.

18. Choi EH, Kim KK, Jun AY, Choi EH, Choi SW, Shin KY. Effects of the off-loading brace on the activation of femoral muscles: a preliminary study. Ann Rehabil Med 2011;35:887-96.

19. Cram JR, Kasman GS, Holtz J. Introduction to surface electromyography. Maryland: Aspen; 1998. p. 360-74.

20. Cholewicki J, VanVliet JJ 4th. Relative contribution of trunk muscles to the stability of the lumbar spine during isometric exertions. Clin Biomech (Bristol, Avon) 2002;17:99-105.

21. Na YM, You SW, Ji SW, Choi HJ. Electromyographic analysis of the concentric \& eccentric contraction in the isokinetic exercise. Korean J Sports Med 2001;19:403-11.

22. Shumway-Cook A, Woollacott M. Motor control : translating research into clinical practice. Philadelphia (PA): Lippincott Williams \& Wilkins; 2001. p. 158-86.

23. Cho MK, Kang JY, Oh JH, Wu JG, Choi EB, Park SE, et al. The effects of performing squats on an inclined board on thigh muscle activation. Phys Ther Rehabil Sci 2017;6:39-44.

24. Nam DH. Effects of squats using visual feedback on the balance ability and thicknesses of quadriceps femoris and transversus abdominis muscle of college students in their 20s [Master thesis]. Deagu: Deagu University; 2017. 